\title{
ULTRASTRUCTURAL CHARACTERIZATION OF THE NEUROMUSCULAR JUNCTION IN DIAPHRAGM OF THE ACETYLCHOLINESTERASE KNOCKOUT MOUSE
}

\author{
*T.A. Hamilton, *R.E. Sheridan, *S.S. Deshpande, **O. Lockridge, and *M. Adler \\ *Comparative Pathology Branch, Comparative Medicine Division, U.S. Army Medical Research \\ Institute of Chemical Defense, Aberdeen Proving Ground, MD, 21010-5400 \\ **Eppley Institute, University of Nebraska Medical Center, Omaha 68198-6805
}

Acetylcholinesterase (AChE) is known to be the primary hydrolyzing enzyme of the neurotransmitter acetylcholine (ACh) at the cholinergic synapses. Recently, questions regarding the role of AChE in the regulation of cholinergic transmission and cholinergic synapse development led to the establishment of an AChE knockout mouse model.[1] The surprising survival of the homozygous AChE defective mutant has led to investigations into possible physiological, biochemical, and morphological compensatory mechanisms. This study set out to characterize the morphology of motor end plates of AChE knockout mouse diaphragm muscle by electron microscopy.

Wild-type and AChE knockout mice, age $58.7 \pm 2.9$ and $46.6 \pm 4.0$ days respectively (mean \pm S.E.), were euthanatized, and diaphragms were removed for morphological studies. Tissue samples were fixed by immersion in buffered $1.6 \%$ formaldehyde $/ 2.5 \%$ glutaraldehyde, followed by routine processing for transmission electron microscopy (JEOL 1200EX). Analyses of motor end plates from AChE knockout mice revealed significant differences in structure when compared with wildtype animals. (FIG.1,2) These observations included alterations to both presynaptic and postsynaptic elements of the end plate. The proximity of terminal axons to their postsynaptic regions was decreased and appeared to contain fewer synaptic vesicles. Junctional folds were fewer in number and irregularly dispersed. Most remarkably, Schwann cells were observed extending into postsynaptic spaces and synaptic clefts. Many of these findings are consistent with morphological changes described in patients with a genetic impairment of collagen-tailed AChE and share some elements in common with chronic experimental auto-induced myasthenia gravis. [2,3]

Research was conducted in compliance with the Animal Welfare Act and other Federal statutes and regulations relating to animals and experiments involving animals and adheres to principles stated in the Guide for the Care and Use of Laboratory Animals, National Research Council, 1996. The facility where this research was conducted is fully accredited by the Association for Assessment and Accreditation of Laboratory Animal Care International. The findings contained herein are the private views of the author(s) and are not to be construed as official or as reflecting the views of the U.S. Army or the Department of Defense.

References

[1] W. Xie et al., Pharm. Exp. Therp. 293(3) (2000) 896-902.

[2] A.G. Engel, Ann. Neurol. 1 (1997) 315.

[3] J.M. Lindstrom, Plasmapheresis and the Immunobiology of Myasthenia Gravis, ed. P.C. Dau, Houghton Mifflin Pub., Boston, MA.,1979 


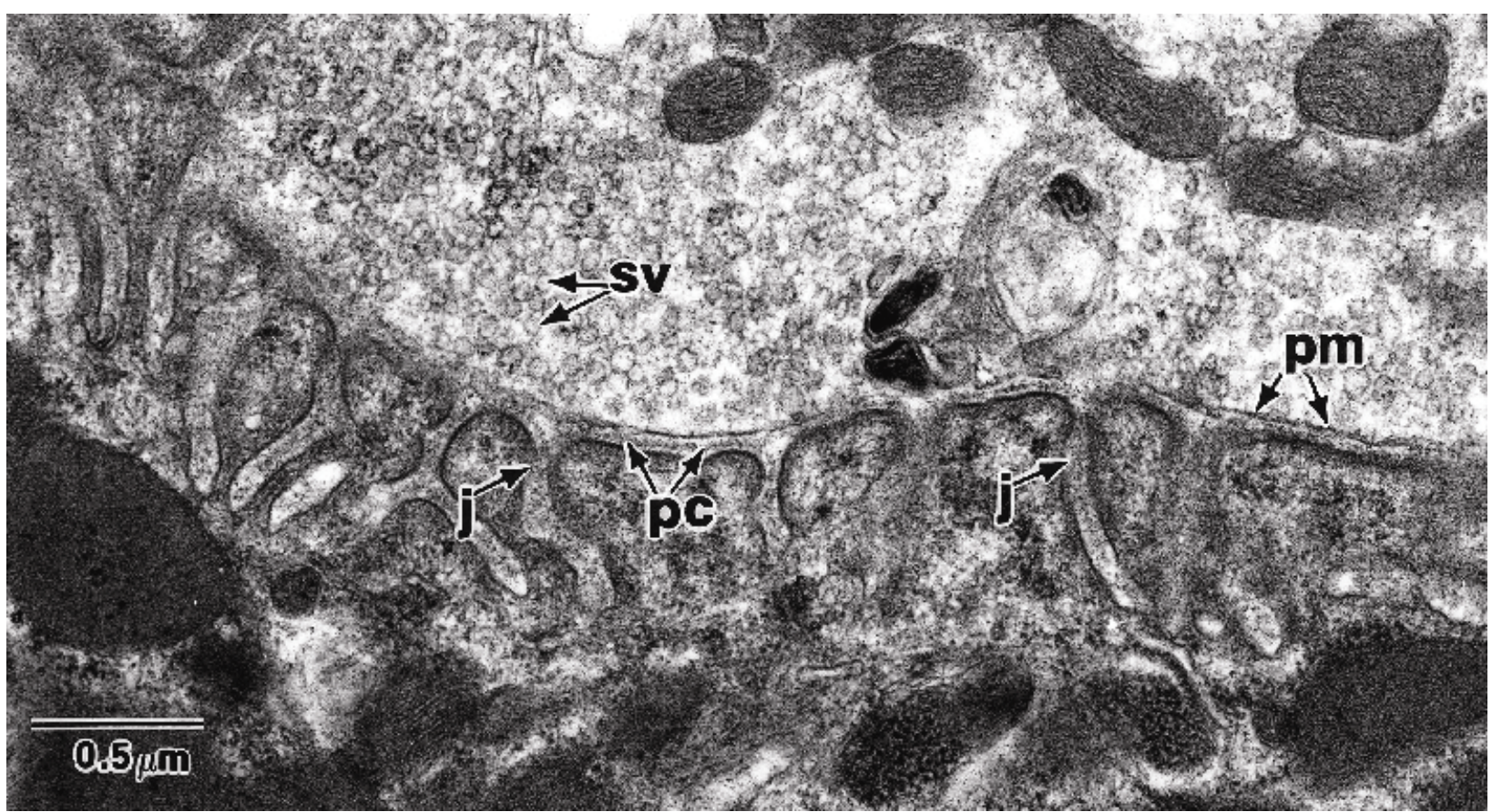

FIG. 1. Motor end plate from diaphragm of wild type mouse. Synaptic vesicles (sv). Presynaptic membrane (pm). Primary cleft (pc). Junctional folds (j).

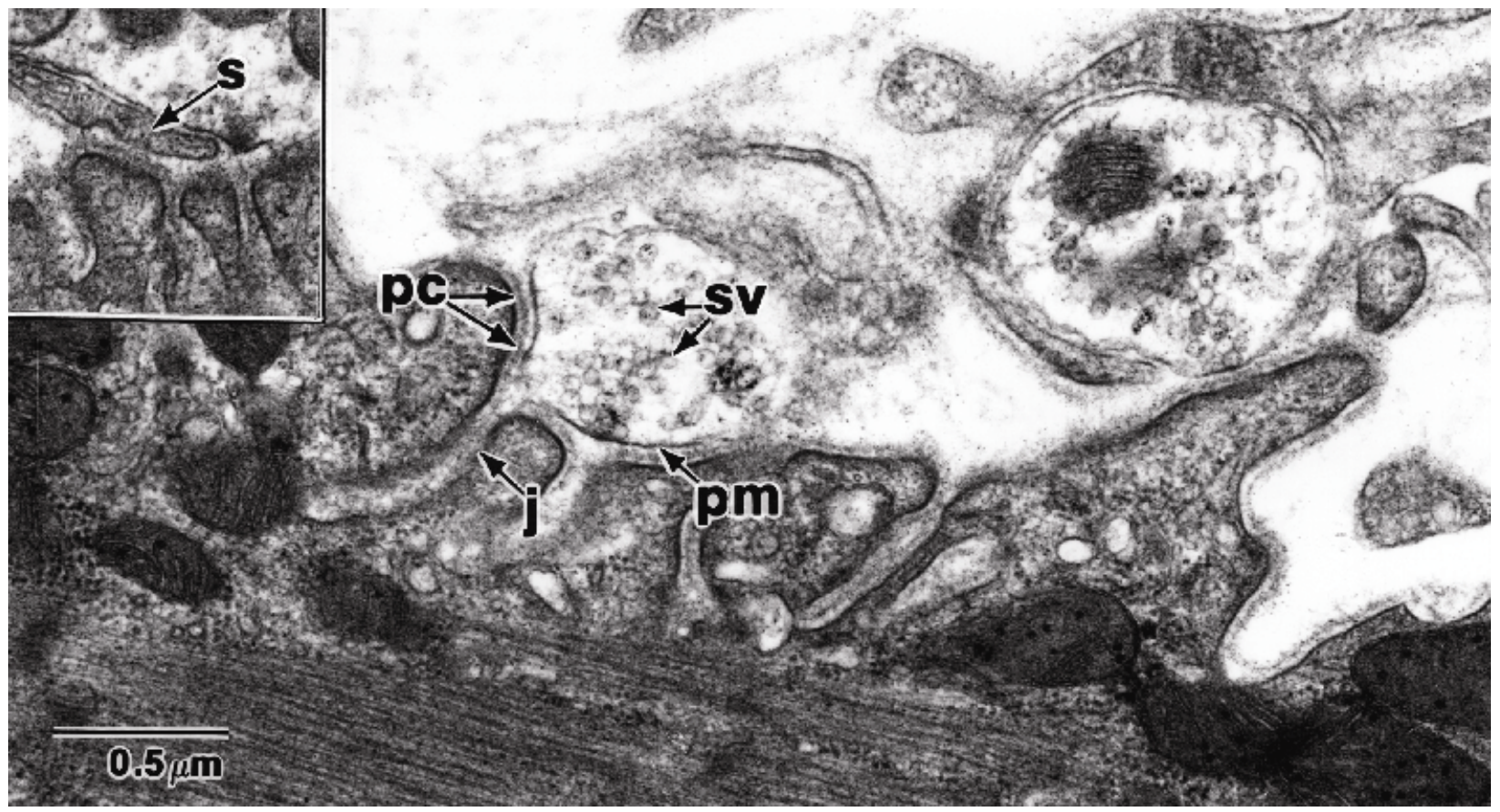

FIG. 2. Motor end plate from diaphragm of AChE knockout type mouse. Synaptic vesicles (sv). Presynaptic membrane (pm). Primary cleft (pc). Junctional fold (j). Schwann cell occupying primary cleft (s). (Inset) 37,000X 